\title{
Modelling and experimental validation of two-dimensional transverse vibrations in a flexible robot link
}

Sørensen, Paul Haase; Baungaard, Jens Rane

Published in:

Proceedings of the IEEE International Conference on Control Applications

Link to article, DOI:

10.1109/CCA.1996.559017

Publication date:

1996

Document Version

Publisher's PDF, also known as Version of record

Link back to DTU Orbit

Citation (APA):

Sørensen, P. H., \& Baungaard, J. R. (1996). Modelling and experimental validation of two-dimensional transverse vibrations in a flexible robot link. In Proceedings of the IEEE International Conference on Control Applications (pp. 942-947). IEEE. https://doi.org/10.1109/CCA.1996.559017

\section{General rights}

Copyright and moral rights for the publications made accessible in the public portal are retained by the authors and/or other copyright owners and it is a condition of accessing publications that users recognise and abide by the legal requirements associated with these rights.

- Users may download and print one copy of any publication from the public portal for the purpose of private study or research.

- You may not further distribute the material or use it for any profit-making activity or commercial gain

- You may freely distribute the URL identifying the publication in the public portal 


\title{
Two-Dimensional Transverse Vibrations in a Flexible Robot Link
}

\author{
Jens Rane Baungaard and Paul Haase Sørensen \\ jrb@iau.dtu.dk $\quad$ phs@iau.dtu.dk \\ Institute of Automation \\ Technical University of Denmark
}

\begin{abstract}
A general model for a rotating homogenous flexible robot link is developed. The model describes twodimensional transverse vibrations induced by the actuator due to misalignment of the actuator axis of rotation relative to the link symmetry axis and due to translational acceleration of the link. The model is validated experimentally.
\end{abstract}

\section{Introduction}

In previously presented work dealing with flexible manipulators, the flexural motion of the links in the vast majority of cases only concerns vibrations in one direction, and those who derive a more universal link model ([4],[5] and [6]) do not investigate the validity of the model in real life experiments.

The general model for a rotating homogenous flexible robot link developed in this paper includes translational acceleration of the link, transverse vibrations in two directions and it handles misalignment of the actuator output shaft relative to the symmetry lines of the link cross section. The link model has been developed in preparation for successive coupling of links, which is helpful in the developing and the design phase of new light weight flexible robots. The effect of misalignment of actuator and robot link in the model is validated experimentally by introducing a known roll angle between actuator shaft and link. The experimental results are compared with simulations and presented in this paper.

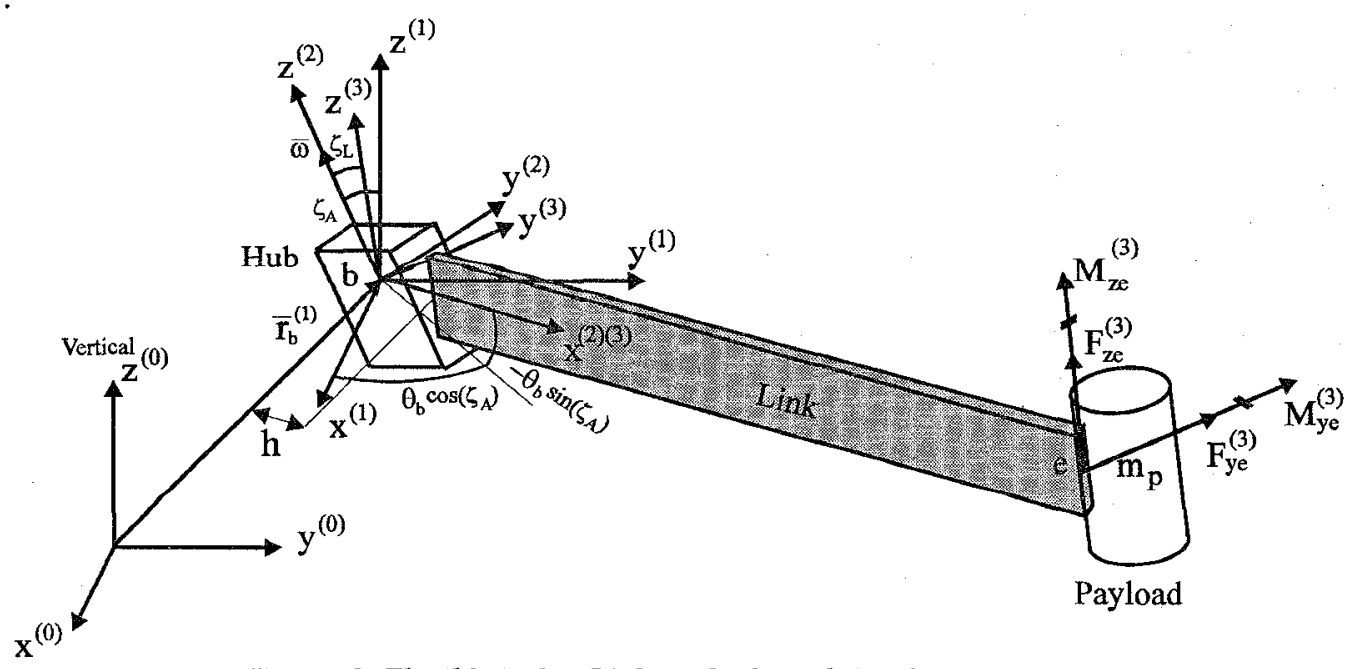

Figure 1. Flexible Robot Link applied the defined frames (0)-(3)
The new extended model has a mechatronic design aspect, so that inaccuracies in the mechanical construction can be simulated, and worst case situations studied in advance, if the accuracy in the mechanical handling and the installation of the robot is known. Simulation results can be used in choosing dimensions of the flexible robot link in order to obtain a certain specified accuracy in positioning the end point.

Turning the argument the other way around, the sphere of possible design solutions for light weight robots, and similarly the possible fields of application for this type of robots have been increased with the extended knowledge about its dynamical behaviour. The results of this paper facilitate a new articulated robot wrist design. Usually, an articulated robot has three degrees of freedom situated in the wrist. This means that the robot must be able to carry the extra weight of transmission and/or actuators. If the first roll motion is moved back to the elbow joint this excess load may be reduced. However, in this case it will be necessary to study two-dimensional transverse vibrations in the outer link.

\section{Modelling}

Before the proper mathematical model is developed and physical considerations are taken, a more detailed description of the link system will be presented and the necessary co-ordinate systems, on which the modelling is based, will be defined. 


\section{Description of the Link System}

Figure 1 shows the rotating homogenous flexible robot link with a payload $m_{p}$ and external forces and torques affecting the link at the end point in $y^{(3)}$ - and $z^{(3)}$ directions: $F_{y e}^{(3)}, F_{z e}^{(3)}, M_{y e}^{(3)}$ and $M_{z e}^{(3)}$. The link is clamped at the base to a rigid hub, which has a spatial dimension and therefore constitutes a rigid connection $h$ between the output shaft and the beginning of the flexible link. The payload is assumed to be rigid and to have the center of mass located on the neutral line of the link at the tip of it.

In addition figure 1 shows a number of chosen coordinate systems used to describe the motion of the link. The frames are related by

Frame $(0)$ is the non-moving base frame.

Frame (1) is frame (0) translated to the point of intersection of the output shaft of the actuator and the neutral line of the link.

Frame (2) is frame (1) rotated so that the $z^{(2)}$-axis points in the direction of the rotation vector $\bar{\omega}$ and the $x^{(2)}$-axis is tangential to the link at the base $(b)$.

Frame (3) is frame (2) rotated around the $x^{(2)}$ axis so that $z^{(3)}$-and $y^{(3)}$-axis follow the symmetric lines of the link.

The link performs a translation described by the relation between frame $(0)$ and frame ( 1$)$

$\overline{\mathrm{r}}_{\mathrm{b}}^{(0)}(\mathrm{t})=\left[\begin{array}{lll}\mathbf{x}_{\mathrm{b}}^{(0)}(\mathrm{t}) & \mathrm{y}_{\mathrm{b}}^{(0)}(\mathrm{t}) & \mathrm{z}_{\mathrm{b}}^{(0)}(\mathrm{t})\end{array}\right]^{\mathrm{T}}$

and a rotation illustrated by the rotation vector $\bar{\omega}$ in figure 1. Index ${ }_{b}$ refers to the hinged end or base of the link. The rotation vector is assumed to lie in the $y^{(3)}-z^{(3)}$ plane and it forms an angle $\varsigma_{A}$ with vertical. In frame (2) the rotation vector is represented as

$\bar{\omega}^{(2)}(\mathrm{t})=\left[\begin{array}{lll}0 & 0 & \dot{\theta}_{\mathrm{b}}(\mathrm{t})\end{array}\right]^{\mathrm{T}}$

The link is assumed to have a roll angle $\varsigma_{L}$ relative to the orientation of the rotation vector around the $x^{(2)}$. axis as indicated in figure 1 .

The flexural vibrations will be modelled with reference to frame (3), but it is easy to transform the motion of the link into anyone of the other mentioned frames given the translational motion eq. (1), its derivative and the rotation vector in eq. (2).

\section{Mathematical Modelling}

The flexible robot link is assumed thin compared to its length and we will assume too that it executes small vibrations. This means that the effects of rotary inertia and shear deformations can be neglected. Furthermore, the link is assumed to be inextensible which is equivalent to saying that the line integral along the link is constant and equal to the length of the link.

We will now take a closer look at the vibrations of the link in figure 2 and introduce the necessary notation.
The motion of a small element of the link which in rest is called $P^{*}$ is described by

$$
\begin{aligned}
& \overline{\mathrm{r}}_{\mathrm{p}^{*}}^{(3)}=\left[\begin{array}{c}
\mathrm{h}+\mathrm{x} \\
0 \\
0
\end{array}\right], \quad \overline{\mathrm{r}}_{\mathrm{p}}^{(3)}=\left[\begin{array}{c}
\mathrm{h}+\mathrm{x}+\mathrm{u}(\mathrm{x}, \mathrm{t}) \\
\mathrm{w}(\mathrm{x}, \mathrm{t}) \\
\mathrm{v}(\mathrm{x}, \mathrm{t})
\end{array}\right] \\
& \text { and } \overline{\mathrm{r}}_{\mathrm{p}}^{(3)}=\overline{\mathrm{r}}_{\mathrm{p}}^{(3)}-\overline{\mathrm{r}}_{\mathrm{p}^{*}}^{(3)}=\left[\begin{array}{c}
\mathrm{u}(\mathrm{x}, \mathrm{t}) \\
\mathrm{w}(\mathrm{x}, \mathrm{t}) \\
\mathrm{v}(\mathrm{x}, \mathrm{t})
\end{array}\right]
\end{aligned}
$$

where $\bar{r}_{p}^{(3)}$ is the position vector and $\bar{l}_{p}^{(3)}$ is the displacement vector of $P$ composed of the displacements $u(x, t), w(x, t)$ and $v(x, t)$ defined in frame (3). $x=0$ corresponds to the clamping position of the link and $x=L$ to the end point of it.

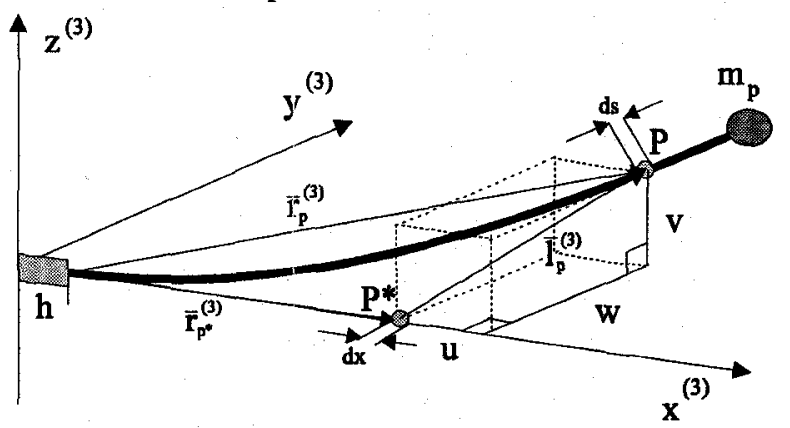

Figure 2. Flexible displacement of the link

The constraint in assuming the link to be inextensible reduces the degrees of freedom for the motion of the link from three to two and the axial motion $u(x, t)$ of an element can be expressed by the two transversal displacements like [8]

$$
u^{\prime}(x, t)=-\frac{1}{2}\left(w^{\prime}(x, t)^{2}+v^{\prime}(x, t)^{2}\right)
$$

The describing partial differential equations (PDE) are developed by use of the Extended Hamilton Principle (EHP) and the system of co-ordinates, that we will refer to, is the translated frame $(1)$.

The EHP is used in the form

$\int_{i_{1}}^{t_{2} L}\left(\delta \hat{\mathrm{T}}_{k}^{(1)}-\delta \hat{\mathrm{V}}^{(1)}+\delta \hat{\mathrm{W}}_{\mathrm{nc}}^{(1)}\right) \mathrm{dxdt}=0$

when $\delta w=\delta v=\delta \theta_{b}=0,0 \leq x \leq L$ at $t=t_{1}$ and $t=t_{2}$.

Here $\delta$ symbolises a virtual displacement, ${ }^{\wedge}$ indicates that it is a density function and $\delta \hat{T}_{k}, \delta \hat{V}$ and $\delta W_{n c}$ are the virtual kinetic, potential and non conservative work density functions, respectively.

\section{Kinetic Energy}

The Kinetic Energy is expressed as

$$
\mathrm{T}_{\mathrm{k}}^{(1)}(\mathrm{t})=\frac{1}{2} \rho \mathrm{A} \int_{0}^{\mathrm{L}} \dot{\overline{\mathrm{r}}}_{\mathrm{p}}^{(1)} \dot{\overline{\mathrm{r}}}_{\mathrm{p}}^{(1)} \mathrm{dx}+\frac{1}{2} \mathrm{~m}_{\mathrm{p}} \dot{\mathrm{r}}_{\mathrm{L}}^{(1)} \dot{\mathrm{r}}_{\mathrm{L}}^{(1)}
$$

where $\rho$ is the mass density for the chosen material and $A$ the cross section area of the link. Index ${ }_{L}$ refers to the function evaluated at the tip $(x=L)$. The velocity of 
element $P$ in frame $(1)$ is found from the position vector in frame (3) (see eq. (3)) by making two rotations and a differentiation

$$
\dot{\overline{\mathrm{r}}}_{\mathrm{p}}^{(1)}=\dot{\mathrm{A}}_{(1.3)}^{-1} \overline{\mathrm{r}}_{\mathrm{p}}^{(3)}+\mathrm{A}_{(1.3)}^{-1} \dot{\overline{\mathrm{r}}}_{\mathrm{p}}^{(3)}=\mathrm{A}_{(1.3)}^{-1}\left[\bar{\omega}^{(3)} \times \overline{\mathrm{r}}_{\mathrm{p}}^{(3)}+\dot{\overline{\mathrm{r}}}_{\mathrm{p}}^{(3)}\right]
$$

The actuator rotation vector $\bar{\omega}$ in frame (3) coordinates is

$$
\begin{aligned}
\bar{\omega}^{(3)}(\mathrm{t}) & =\mathrm{A}_{(2.3)} \bar{\omega}^{(2)}(\mathrm{t})=\left[\begin{array}{lll}
0 & \dot{\theta}_{\mathrm{b}}(\mathrm{t}) \mathrm{a}_{\mathrm{A}} & \dot{\theta}_{\mathrm{b}}(\mathrm{t}) \mathrm{b}_{\mathrm{A}}
\end{array}\right]^{\mathrm{T}} \\
\mathrm{a}_{\mathrm{A}} & =\sin \left(\zeta_{\mathrm{L}}\right), \mathrm{b}_{\mathrm{A}}=\cos \left(\zeta_{\mathrm{L}}\right)
\end{aligned}
$$

$A_{(i, j)}$ is the transformation matrix from frame $(i)$ to frame $(j)$.

Substituting eq. (3) and (8) into equation eq.(7) and using the expression for the kinetic energy eq.(6) gives after a number of manipulations and retaining only terms of less than second order

$$
\begin{aligned}
& \mathrm{T}_{\mathrm{k}}^{(1)}=\frac{1}{2} \int_{0}^{\mathrm{L}}\left(\rho \mathrm{A}+\mathrm{m}_{\mathrm{p}} \delta(\mathrm{x}-\mathrm{L})\right) \\
& {\left[\dot { \theta } _ { \mathrm { b } } ^ { 2 } \left(\mathrm{a}_{\mathrm{A}}{ }^{2}\left(\mathrm{v}^{2}+(\mathrm{h}+\mathrm{x})^{2}\right)+\mathrm{b}_{\mathrm{A}}{ }^{2}\left(\mathrm{w}^{2}+(\mathrm{h}+\mathrm{x})^{2}\right)\right.\right.} \\
& \left.-2 \mathrm{a}_{\mathrm{A}} \mathrm{b}_{\mathrm{A}} \mathrm{vw}\right)-2 \dot{\theta}_{\mathrm{b}}\left(\mathrm{a}_{\mathrm{A}} \dot{\mathrm{v}}(\mathrm{h}+\mathrm{x})-\mathrm{b}_{\mathrm{A}} \dot{\mathrm{w}}(\mathrm{h}+\mathrm{x})\right) \\
& \left.+\left(\dot{\mathrm{w}}^{2}+\dot{\mathrm{v}}^{2}\right)\right] \mathrm{dx}-\frac{1}{2} \dot{\theta}_{\mathrm{b}}^{2} \int_{0}^{\mathrm{L}}\left[\mathrm{f}(\mathrm{x})\left(\mathrm{w}^{\prime 2}+\mathrm{v}^{\prime 2}\right)\right] \mathrm{dx}
\end{aligned}
$$

The longitudinal motion $u(x, t)$ has been eliminated by introducing the dependency in eq.(4) which has introduced the function

$$
f(x)=\int_{h+x}^{h+L}\left(\rho A+m_{p} \delta(\xi-L)\right) \xi d \xi
$$

where $\delta()$ is the Dirac Delta function.

\section{Potential Energy}

The potential energy consists of two strain energy terms from the bending in two planes, the contributions due to gravity and the translational accelerations of the translated frame $(1)$ relative to the base frame $(0)$

$$
\begin{aligned}
& \bar{a}_{b}^{(0)}=\left[\begin{array}{lll}
\ddot{x}_{b}^{(0)} & \ddot{y}_{b}^{(0)} & \ddot{z}_{b}^{(0)}
\end{array}\right]^{T} \\
& \bar{a}_{b g}^{(1)}=\left[\begin{array}{lll}
-\ddot{x}_{b}^{(0)} & -\ddot{y}_{b}^{(0)} & -\ddot{z}_{b}^{(0)}-g
\end{array}\right]^{T}
\end{aligned}
$$

The translational acceleration can be interpreted as a time varying fictive gravity in frame (3) and in the expression for the potential energy

$$
\begin{aligned}
V^{(1)} & =\frac{1}{2} \int_{0}^{L}\left(E I_{z} w^{\prime 2}+E I_{y} v^{\prime \prime 2}\right. \\
& \left.+2\left(\rho A+m_{p} \delta(x-L)\right)\left[\bar{a}_{b g}^{(1) T} A_{(1.2)}^{-1} A_{(2.3)}^{-1} \bar{r}_{p}^{(3)}\right]\right) d x \\
& =\frac{1}{2} \int_{0}^{L}\left(E I_{z} w^{\prime \prime 2}+E I_{y} v^{\prime \prime 2}+2\left(\rho A+m_{p} \delta(x-L)\right) *\right. \\
& {\left.\left[a_{b g}^{(3)}\left(\theta_{b}, t\right) u+a_{b g}^{(3)}\left(\theta_{b}, t\right) w+a_{b g}^{(3)}\left(\theta_{b}, t\right) v\right]\right) d x }
\end{aligned}
$$

The rotations $A_{(1.2)}^{-1}$ and $A_{(2,3)}^{-1}$ are trigonometric functions of the constant roll angle $\varsigma_{L}$, output shaft angle $\varsigma_{A}$ and the variable rotation angle $\theta_{b}$.

$a_{b g i}^{(3)}\left(\theta_{b}, t\right) \quad i=1,2,3$ is the $i$ th element of the acceleration vector $\bar{a}_{b g}^{(3)}\left(\theta_{b}, t\right)$. The point of zero potential energy is defined by $\theta_{b}=0, w=0, v=0$ and $u=0$. The insignificant contribution from the longitudinal displacement $u$ to the potential energy will be ignored in the following.

\section{Non Conservative Work}

The non conservative work $W_{n c}$ is the elementary work of non potential specified forces. In this case we actuate the link by a torque $T_{e x t}$ from an actuator at the hub end $b$. Furthermore the description includes external forcing terms at the tip point (see figure 1), which can arise for instance if the flexible robot link has contact to the environment and is used in force control or due to reactions from a subsequent link. The total nonconservative work becomes

$$
\begin{aligned}
& \mathrm{W}_{\mathrm{nc}}^{(1)}=\int_{0}^{\mathrm{L}} \hat{\mathrm{W}}_{\mathrm{nc}}^{(1)} \mathrm{dx} \\
& =\mathrm{T}_{\mathrm{ext}} \theta_{\mathrm{b}}+\left(\mathrm{F}_{\mathrm{ye}}^{(3)} \mathrm{W}+\mathrm{F}_{\mathrm{ze}}^{(3)} \mathrm{v}\right) \delta(\mathrm{x}-\mathrm{L}) \\
& +\left(\mathrm{M}_{\mathrm{ze}}^{(3)} \mathrm{W}^{\prime}+\mathrm{M}_{\mathrm{ye}}^{(3)} \mathrm{v}^{\prime}\right) \delta(\mathrm{x}-\mathrm{L})
\end{aligned}
$$

Substituting (9), (12) and (13) into EHP gives

$$
\begin{aligned}
& \int_{t_{1}}^{t_{2}} \int_{0}^{L}\left\{\left(\rho A+m_{p} \delta(x-L)\right)\right. \\
& {\left[\dot { \theta } _ { b } \delta \dot { \theta } _ { b } \left(a_{A}^{2}\left(v^{2}+(h+x)^{2}\right)+b_{A}^{2}\left(w^{2}+(h+x)^{2}\right)\right.\right.} \\
& \left.\quad-2 a_{A} b_{A} v w\right) \\
& +\dot{\theta}_{b}^{2}\left(a_{A}^{2} v \delta v+b_{A}^{2} w \delta w-a_{A} b_{A} w \delta v-a_{A} b_{A} v \delta w\right) \\
& -\delta \dot{\theta}_{b}\left(a_{A} \dot{v}(h+x)-b_{A} \dot{w}(h+x)\right) \\
& \left.-\dot{\theta}_{b}\left(a_{A}(h+x) \delta \dot{v}-b_{A}(h+x) \delta \dot{w}\right)+\dot{w} \delta \dot{w}+\dot{v} \delta \dot{v}\right] \\
& -\dot{\theta}_{b} \delta \dot{\theta}_{b}\left[f(x)\left(w^{\prime 2}+v^{\prime 2}\right)\right]-\dot{\theta}_{b}^{2}\left[f(x)\left(w^{\prime} \delta w^{\prime}+v^{\prime} \delta v^{\prime}\right)\right]
\end{aligned}
$$

$$
-\left(E I_{z} w^{\prime \prime} \delta w^{\prime \prime}+E I_{y} v^{\prime \prime} \delta v^{\prime \prime}+\left(\rho A+m_{p} \delta(x-L)\right)\right.
$$$$
\left(\left[\frac{d\left(a_{t 1}\left(\theta_{b}, \bar{a}_{b g}^{(0)}\right)\right)}{d \theta_{b}}(x+h)+\frac{d\left(a_{t 2}\left(\theta_{b}, \bar{a}_{b g}^{(0)}\right)\right)}{d \theta_{b}} w\right.\right.
$$$$
\left.+\frac{d\left(a_{t 3}\left(\theta_{b}, \bar{a}_{b g}^{(0)}\right)\right)}{d \theta_{b}} v\right] \delta \theta_{b}
$$

$$
\left.\left.+\left[a_{t 2}\left(\theta_{b}, \bar{a}_{b g}^{(0)}\right) \delta w+a_{t 3}\left(\theta_{b}, \bar{a}_{b g}^{(0)}\right) \delta v\right]\right)\right)
$$


$+T_{e x t} \delta \theta_{b}+\left(F_{y e}^{(3)} \delta w+F_{z e}^{(3)} \delta v\right) \delta(x-L)$

$\left.+\left(M_{z e}^{(3)} \delta w^{\prime}+M_{y e}^{(3)} \delta v^{\prime}\right) \delta(x-L)\right\} d x d t=0$

when $\delta w=\delta v=\delta \theta_{b}=0,0 \leq x \leq L$ at $t=t_{1}$ and $\mathrm{t}=t_{2}$

Suitable integrating by part with respect to time $t$ or the spatial variable $x$ results in the following PDEs.

Variations taken with respect to: $w_{,} w^{\prime}, w^{\prime \prime}$ and $\dot{w}$

$$
\begin{aligned}
& E I_{z} w^{(4)}+\rho A \ddot{w}= \\
& -\left(\rho A+m_{p} \delta(x-L)\right) \ddot{\theta}_{b} b_{A}(h+x) \\
& -m_{p} \delta(x-L) \ddot{w} \\
& +\dot{\theta}_{b}^{2}\left[\left(\rho A+m_{p} \delta(x-L)\right)\left(b_{A}^{2} w-a_{A} b_{A} v\right)\right. \\
& \left.+f^{\prime}(x) w^{\prime}+f(x) w^{\prime \prime}\right] \\
& -\left(\rho A+m_{p} \delta(x-L)\right) a_{b_{g} 2}^{(3)}\left(\theta_{b}, t\right) \\
& +F_{y e}^{(3)} \delta(x-L)-M_{z e}^{(3)} \delta^{\prime}(x-L)
\end{aligned}
$$

with the corresponding boundary conditions at $x=0, L$

either $E I_{z} w^{\prime \prime \prime}=\dot{\theta}_{b}^{2} f(x) w^{\prime}$ or $w=0$

and

either $w^{\prime \prime}=0$ or $w^{\prime}=0$

Because we have the interpretation that the link is Clamped-Free the geometric boundaries at $x=0$ and the dynamic boundaries at $x=L$ become

$w(0, t)=0$ and $w^{\prime}(0, t)=0$

$w^{\prime \prime}(L, t)=0$ and $w^{\prime \prime \prime}(L, t)=0$

notice from (10) that $f(L)=0$

Variations taken with respect to: $v, v^{\prime}, v^{\prime \prime}$ and $\dot{v}$

In consequence of the symmetry in $v$ and $w$ in eq. (14) the result follows from above by substituting:

- $w$ and its derivatives by expressions in $v$.

- the flexural rigidity $E I_{z}$ in the $y^{(3)}$-direction is substituted with the $z^{(3)}$-direction rigidity $E I_{y}$.

- external forces $F_{y e}^{(3)}, M_{y e}^{(3)}$ by the corresponding quantities acting in $z^{(3)}$-direction $F_{z e}^{(3)}, M_{z e}^{(3)}$.

- Because it is the translational acceleration in the $z^{(3)}$-direction that has influence on $v, a_{b g 2}^{(3)}\left(\theta_{b}, t\right)$ are replaced by $a_{b b^{3}}^{(3)}\left(\theta_{b}, t\right)$.

- the projection $a_{A}$ is exchanged with $b_{A}$ and vice versa.

- The sign of the first term on the right hand side of (15) changes.
The equation relating the interaction between the actuator torque $T_{e x t}$ and link reactions is found by taking variations with respect to $\theta_{b}$ and $\dot{\theta}_{b}$

\section{Solutions to the PDEs}

Due to the analogy between the flexural vibrations $v$ and $w$ described earlier we restrict the following to the PDE, handling the $w$ vibrations.

The solution to the PDE eq.(15) is separated in space and time. The separation method involves the expansion

$$
w(x, t)=\sum_{j=1}^{\infty} \varphi_{j}(x) q_{j}(t) \wedge v(x, t)=\sum_{j=1}^{\infty} \varphi_{j}(x) p_{j}(t)
$$

where $q_{i}$ and $p_{i}$ are the respectively time dependent function in the expansion of $w$ and $v$. The mode shape functions $\varphi_{j}(x)$ satisfy the boundary conditions eq.(18) and they are chosen so that they are orthogonal and normalised according to

$$
\begin{aligned}
& \int_{0}^{\mathrm{L}} \varphi_{\mathrm{j}}(\mathrm{x}) \varphi_{\mathrm{i}}(\mathrm{x}) \mathrm{dx}=0 \quad \text { for } \mathrm{i} \neq \mathrm{j} \\
& \text { and } \varphi_{\mathrm{j}}(\mathrm{L})=(-1)^{(\mathrm{j}+1)} \quad \forall \mathrm{j}
\end{aligned}
$$

Substituting the expansion eq.(19) into the PDE eq.(15), and multiplying by $\varphi_{i}(x)$ and using the orthogonality of the mode shape functions gives the ordinary differential equation (ODE)

$$
\begin{aligned}
& \frac{E I_{z}}{\rho A} \gamma_{i}^{4} q_{i}+\ddot{q}_{i}=\ddot{\theta}_{b} b_{A} \alpha_{i}+\sum_{j=1}^{\infty} \beta_{i j} \ddot{q}_{j} \\
& +\dot{\theta}_{b}^{2}\left[\left(1+\sum_{j=1}^{\infty} \beta_{i j}\right)\left(b_{A}^{2} q_{i}-a_{A} b_{A} p_{i}\right)+\sum_{j=1}^{\infty} \kappa_{i j} q_{j}\right] \\
& +a_{b g}^{(3)}\left(\theta_{b}, t\right) \beta_{i}+F_{y e}^{(3)} \frac{\varphi_{i}(L)}{\mu}-M_{z e}^{(3)} \frac{\varphi_{i}^{\prime}(L)}{\mu}
\end{aligned}
$$

where

\begin{tabular}{|l|c|c|c|c|}
\hline modeNo. & 1 & 2 & 3 & 4 \\
\hline$\gamma_{i} L$ & 1.875 & 4.694 & 7.855 & 11.00 \\
\hline
\end{tabular}

The various new constants are

$$
\begin{aligned}
& \mu=\rho A \int_{0}^{L} \varphi_{i}^{2} d x=\frac{\rho A L}{4} \\
& \alpha_{i 0}=-\frac{\rho A}{\mu} \int_{0}^{L}(h+x) \varphi_{i} d x \\
& \alpha_{i}=\alpha_{i 0}-\frac{m_{p}(h+L) \varphi_{i}(L)}{\mu} \\
& \beta_{i 0}=\frac{\rho A}{\mu} \int_{0}^{L} \varphi_{i} d x \quad, \quad \beta_{i}=\beta_{i 0}-\frac{m_{p}}{\mu} \varphi_{i}(L)
\end{aligned}
$$




$$
\begin{aligned}
& \beta_{i j}=\frac{m_{p}}{\mu} \varphi_{i}(L) \varphi_{j}(L) \\
& \kappa_{i j}=-\frac{\rho A}{2 \mu} \int_{0}^{L}\left((h+L)^{2}-(h+x)^{2}\right) \varphi_{j}^{\prime} \varphi_{i}^{\prime} d x-\frac{m_{p} L}{\mu} \varphi_{j}^{\prime}(L) \varphi_{i}^{\prime}(L)
\end{aligned}
$$

So far we have not been concerned with the dissipation of energy due to natural damping of the vibrations in the link. If the dampings are assumed to have viscous nature [3] an extra term must be added on the left of eq. (15). If we find the solution for all $i$ with a viscous damping term included and gather the results in one ODE we find the final solution to the PDE eq.(15)

$$
\begin{aligned}
& \overline{\ddot{q}}+R_{w} \overline{\dot{q}}+W_{w} \bar{q}= \\
& \ddot{\theta}_{b} b_{A} \bar{\alpha}+G \overline{\ddot{q}}+\dot{\theta}_{b}^{2}\left[H\left(b_{A}^{2} \bar{q}-a_{A} b_{A} \bar{p}\right)+K \bar{q}\right] \\
& +a_{b g}^{(3)}\left(\theta_{b}, t\right) \bar{d}_{1}+F_{y e}^{(3)} \bar{d}_{2}-M_{z e}^{(3)} \bar{d}_{3}
\end{aligned}
$$

where

$$
\begin{aligned}
& \mathrm{R}_{\mathrm{w}}=\operatorname{diag}_{\mathrm{i}}\left[2 \varsigma_{\mathrm{wi}} \omega_{\mathrm{wi}}\right] \quad \mathrm{W}_{\mathrm{w}}=\operatorname{diag}_{\mathrm{i}}\left[\omega_{\mathrm{wi}}^{2}\right] \\
& \bar{\alpha}=\left[\begin{array}{c}
\alpha_{1} \\
\alpha_{2} \\
\vdots
\end{array}\right] \quad \mathrm{G}(\mathrm{i}, \mathrm{j})=\frac{\mathrm{m}_{\mathrm{p}}}{\mu} \varphi_{\mathrm{i}}(\mathrm{L}) \varphi_{\mathrm{j}}(\mathrm{L})=\frac{\mathrm{m}_{\mathrm{p}}}{\mu}(-1)^{\mathrm{i}+\mathrm{j}} \\
& \mathrm{H}=\operatorname{diag}_{\mathrm{i}}\left[1+\sum_{\mathrm{j}=1}^{\infty} \beta_{\mathrm{ij}}\right] \quad \mathrm{K}(\mathrm{i}, \mathrm{j})=\kappa_{\mathrm{ij}} \\
& \overline{\mathrm{d}}_{1}=\left[\begin{array}{c}
\beta_{1} \\
\beta_{2} \\
\vdots
\end{array}\right] \overline{\mathrm{d}}_{2}=\frac{1}{\mu}\left[\begin{array}{c}
\varphi_{1}(\mathrm{~L}) \\
\varphi_{2}(\mathrm{~L}) \\
\vdots
\end{array}\right] \quad \overline{\mathrm{d}}_{3}=\frac{1}{\mu}\left[\begin{array}{c}
\varphi_{1}^{\prime}(\mathrm{L}) \\
\varphi_{2}^{\prime}(\mathrm{L}) \\
\vdots
\end{array}\right]
\end{aligned}
$$

Term 3 in the right side of eq.(23) represents the nonlinear centrifugal effects and constitutes the coupling between the two transverse vibrations via the coupling matrix $H$.

Similar results are found for the vibrations $v$ and because the mode shape functions are the same in the two directions only the matrices $R_{v}$ and $W_{v}$ in eq(24) change.

\section{Experimental Validation}

\section{Description of the experimental set-up}

The experimental set-up used to validate the model consists of a DC-motor with an analog tacho feedback. On the output shaft of the motor there is a backlash free gear and on the load side of the gear the hub and Link follow. The link is an aluminium beam with dimensions $1200 \times 40 \times 2$ [mm]. Two pairs of Strain Gauges (SG) are used as sensors to measure the vibrations in each vibration plane. With two sets of SGs it is possible to measure the first two modes $q_{1}$ and $q_{2}$ and the contribution from the residual modes to the sensor signals are considered as noise. The placement of the SG sensors are chosen so that the sensor signals do not obtain contributions from the 3rd mode which is the residual mode containing most energy. In addition to
SGs the sensor system consists of a potentiometer mounted directly on the output shaft to measure $\theta_{b}$.

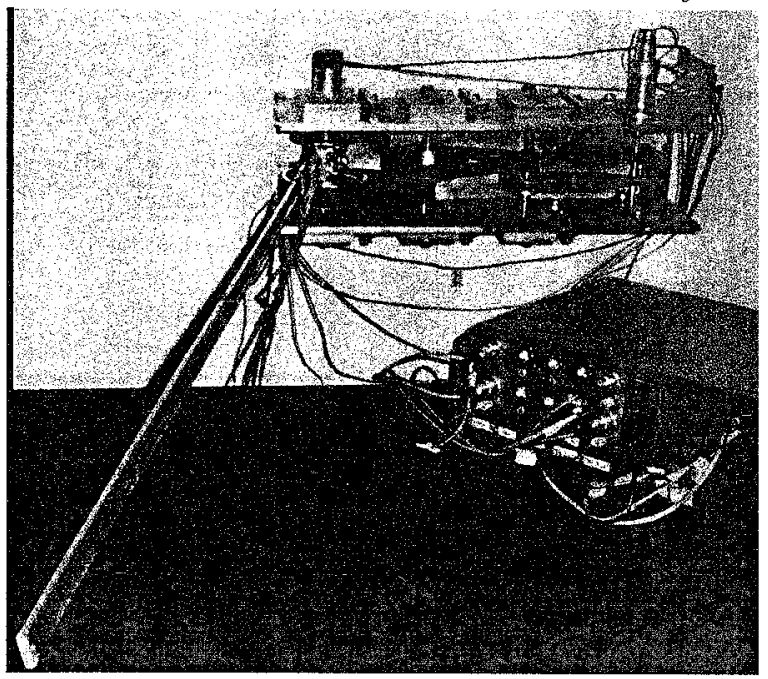

Figure 3. Flexible I DOF Robot

\section{Experiments}

The actuator model is of 2 nd order and the flexural vibration model used in the design of a controller is the linearized version of eq.(23) truncated to two modes i.e. the third term on the right which represents the centrifugal contribution of the motion is neglected. We end up having a state space model of order six with the states

$$
\overline{\mathrm{x}}=\left[\begin{array}{llllll}
\theta_{\mathrm{b}} & \dot{\theta}_{\mathrm{b}} & \mathrm{q}_{1} & \mathrm{q}_{2} & \dot{\mathrm{q}}_{1} & \dot{\mathrm{q}}_{2}
\end{array}\right]^{\mathrm{T}}
$$

The system is augmented with an extra integral state to take care of non-linear effects like stiction and coulomb friction in the actuator.

The derivatives in eq.(25) are not measurable, consequently a Discrete Predictive Kalmanfilter was used. Subsequently a LQG controller was designed and implemented. The hub position was weighted far the most in order to obtain highest speed $\dot{\theta}_{b}$ and to see some vibrations that could be compared with corresponding simulations of the non-linear system eq.(23).

Experiment 1: $\varsigma_{A}=0^{\circ}, \varsigma_{L}=0^{\circ}$ and $m_{p}=0[\mathrm{~kg}]$

The controller was designed for the system with no payload and where $\varsigma_{A}=0$ and $\varsigma_{L}=0$ i.e. a system where the control signal is determined on basis of sensor signals measuring in the same plane as the applied actuator torque $T_{e x t}$. The controlled variable in this system is the angle at the end point of the link which is determined by dead reckoning

$$
\theta_{\mathrm{e}}(\mathrm{t})=\theta_{\mathrm{b}}(\mathrm{t})+\mathrm{q}_{1}(\mathrm{t}) \varphi_{1}^{\prime}(\mathrm{L})+\mathrm{q}_{2}(\mathrm{t}) \varphi_{2}^{\prime}(\mathrm{L})
$$

The experiment is carried out with a step respons of 0.3 [rad] at $t=O[\mathrm{~s}]$ as reference signal. Almost no vibrations in the plane perpendicular to the plane of rotation were seen. The maximum observed amplitude of $p_{1}$ was of magnitude $2 e-4[\mathrm{~m}]$. 


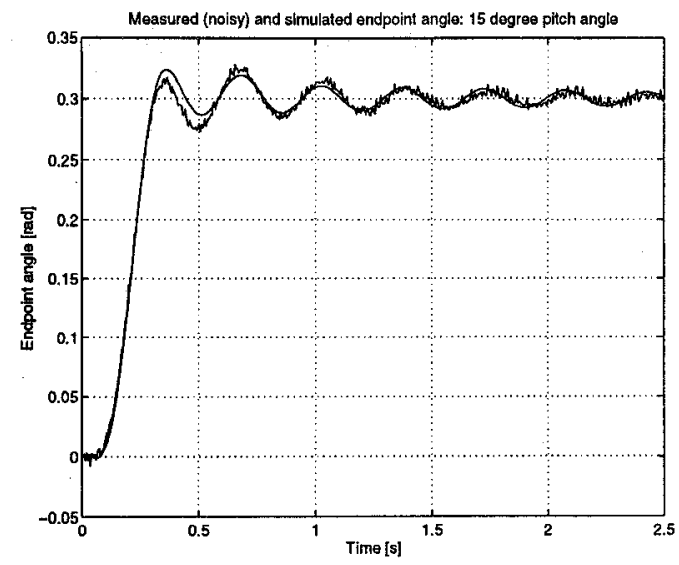

Figur 4. Measured (noisy) and simulated end point angle.

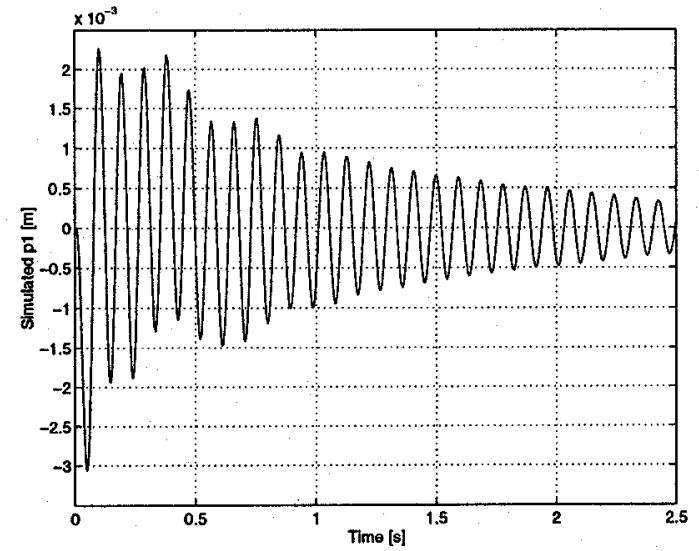

Figur 5. Simulated 1st. mode $p_{1}$ of the out-of-plane vibrations.

Experiment 2: $\varsigma_{A}=0^{\circ}, \varsigma_{L}=15^{\circ}$ and $m_{p}=0 .[\mathrm{kg}]$

The only change in the experimental set-up, in relation to the first experiment, is a change in the roll angle of the link to $15^{\circ}$. The two plots of the measured and simulated $p_{1}$ show very fine agreement with respect to both amplitude level, frequency and damping. The superimposed vibration seen in the measured $p_{I}$ is caused by inaccurate sensor location and uncertainties in the determination of the amplification of the SGsignal. Both uncertainties affect the elements in the decoupling matrix.

\section{Conclusions}

The describing PDEs for a rotating and translating flexible robot link with misaligned actuator and link symmetry lines were derived by the use of the Extended Hamilton Principle and they were solved by separation of variables. The solution is a system of an infinite number of ODEs represented in eq.(23)(24), and we found that the differential equations are highly nonlinear and that the flexural vibrations $\mathrm{w}$ and $v$ are coupled through the matrix $H$.

Experiments compared with corresponding simulations of the link model verify the effect of misalignment of actuator and link.

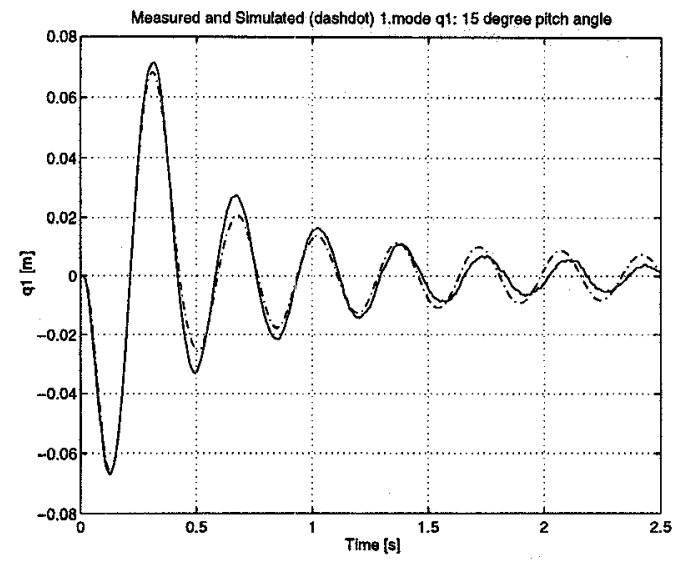

Figur 6. Measured and simulated (dash-and-dot) 1st. mode $q_{1}$.

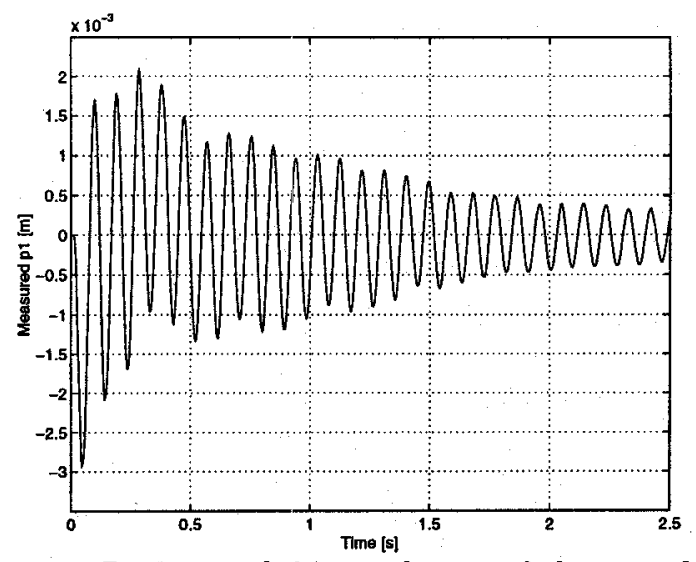

Figur 7. Measured 1st: mode $p_{1}$ of the out-of-plane vibrations.

\section{References}

[1] H.Asada and Z.-D. Ma, "Inverse Dynamics of Flexible Robot Arms - Part I: Modelling, Linearization and Analysis", v 11, ASME, New York, USA. pp.45$60,1988$.

[2] G. Christensen, E. Both and P.Ø. Sørensen, "Mekanik", Lab. for Teknisk Fysik 1990 ( in Danish)

[3] R.W. Clough and J. Penzien, "Dynamics of Structures", McGraw-Hill 1975

[4] V.E. Berbyuk and M.V. Demidyuk, "Controlled Motion of an Elastic Manipulator with Distributed Parameters", Mekhanika Tverdogo Tela, Vol.19 No.2, pp. 59-67, 1984

[5] W.J. Book, "Recursive Lagranian Dynamics of Flexible Manipulator Arms" International Journal of Robotic Research Vol. 3, pp. 198-205, 1986

[6] C.-J. Li and T.S. Sankar, "A Systematic Method of Dynamics for Flexible Robot Manipulators". Journal of Robotic Systems 9(7), pp.861-891, 1992

[7] L.Meirovitch, "Analytical Methods in Vibrations", MacMillan, New York 1967

[8] L.Meirovitch, "Computational Methods in Structural Dynamics", Sijthoff \& Noordhoff 1980 